\title{
Morphology of the sinus node in human and mouse hearts with isomerism of the atrial appendages
}

Siew Yen Ho, Jeong-Wook Seo, Nigel A Brown, Andrew C Cook, Nuala L K Fagg, Robert H Anderson

\begin{abstract}
Background-The location of the sinus node is known to be at best abnormal, or at worst unknown, in patients with isomerism of the morphologically left atrial appendage. In contrast, the sinus node is known to be an excellent histological marker of the morphologically right appendage, being duplicated in those with right isomerism. The aim of the study was to investigate this condition further in fetal human and mouse hearts.
\end{abstract}

Methods-Serial histological sections of the area anticipated to contain the sinus node were studied in hearts with isomerism of the atrial appendages taken from 14 human fetuses and 13 ivliv mice, using 12 mouse hearts with normally arranged or mirror imaged atrial chambers for controls.

Results-All hearts with isomerism of the right appendages (two human and four mouse) had bilateral sinus nodes. The cases with isomerism of the left appendages (12 human and nine mouse) showed absence of a recognisable sinus node except in four cases $(19 \%)$ in which a small remnant of the node was found. In three of these cases, it was related postero-inferiorly to the superior cavoatrial junction.

Conclusions-The concept of isomerism of the atrial appendages is endorsed by findings on the morphology of the sinus node, this being the most reliable histological criterion for existence of a morphologically right atrium. A small proportion of hearts with left isomerism had a structure resembling the sinus node, but it was hypoplastic and displaced postero-inferiorly, distant from its expected position had the hearts possessed an incompletely formed morphologically right appendage.

(Br Heart f 1995;74:437-442)

Keywords: sinus node morphology; isomerism of atrial appendages; situs inversus; conduction system

Since the introduction of the concept of isomerism to describe the morphology of hearts with the so called "heterotaxic syndrome", arguments have continued as to whether isomerism is really present or whether the constellation of lesions would better be understood in terms of partial expression of either the usual or mirror imaged atrial arrangements. ${ }^{23}$ One of the ways to confirm the presence of an isomeric arrangement would be to provide morphological proof in such cases of the presence of a histologically recognisable sinus node.

The sinus node in the human is a distinct and obvious anatomical structure found subepicardially at the junction between the venous component and the appendage of the morphologically right atrium. ${ }^{4}$ The node is functionally important in initiating the heart beat, which begins very early in life and continues for a long period without changing its biological and physiological characteristics. ${ }^{5}$ It may reasonably be presumed that the sinus node is one of the original anatomical features of the morphologically right atrium, and is unlikely to be affected by lesions involving its venous component. With this premise in mind, we searched histologically for the sinus node in human fetal hearts which were part of a series recognised morphologically as having an isomeric arrangement of the atrial appendages. ${ }^{6}$ We have also previously identified a group of $i v / i v$ mouse hearts with isomeric arrangement of the atrial appendages.? Since the mouse heart is small enough to be readily studied in its entirety by histological techniques, we supplemented the human study with murine hearts to determine whether the sinus node is also a marker of right atrial morphology in the mouse.

\section{Methods}

We studied 14 human hearts ranging from 12-25 weeks gestation, and from newborn to one week in age. These formed part of a study group reported previously. ${ }^{68}$ Left isomerism was recognised when both atrial appendages were finger-like in shape and had narrow junctions with the venous component of the atria. In addition, pectinate muscles were confined within the atrial appendage. In contrast, both atrial appendages were triangular in shape with broad junctions in hearts with right isomerism. Furthermore, the pectinate muscles which radiate from the terminal crest extended around the vestibule of the atria to meet at the crux. Using these criteria, 12 of these fetal hearts had isomerism of the morphologically left atrial appendages, while the remaining two had isomerism of the morphologically right appendages.

Twenty five $i v / i v$ mice of the SI/col background, originally from the Jackson Laboratory (Bar Harbour, USA), were also 


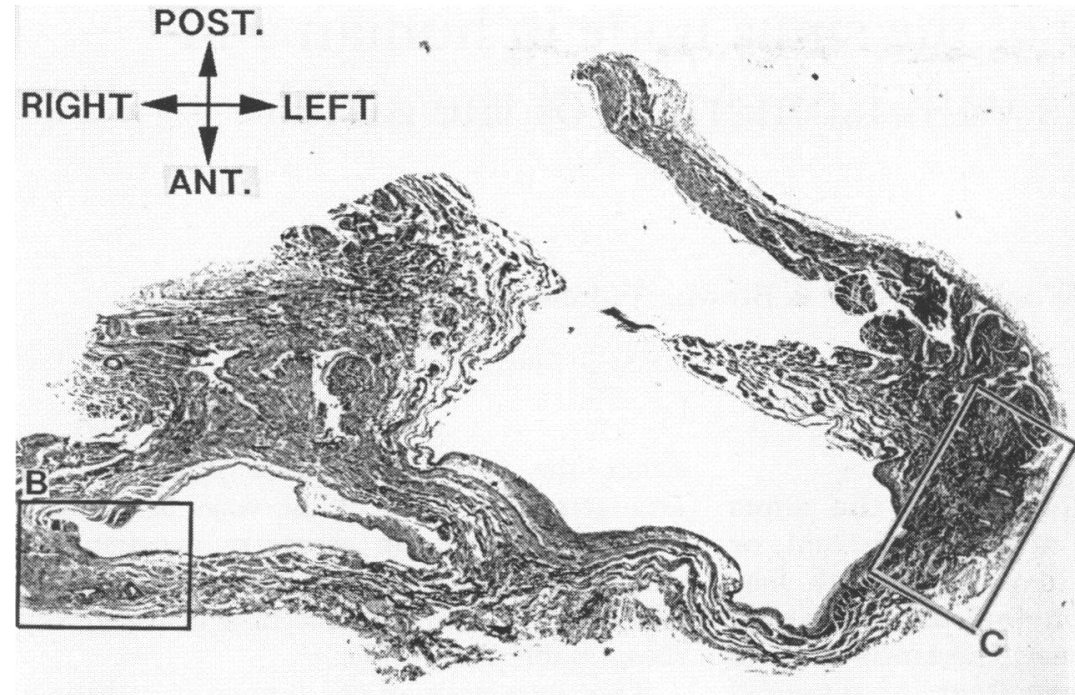

A $1 \mathrm{~mm}$
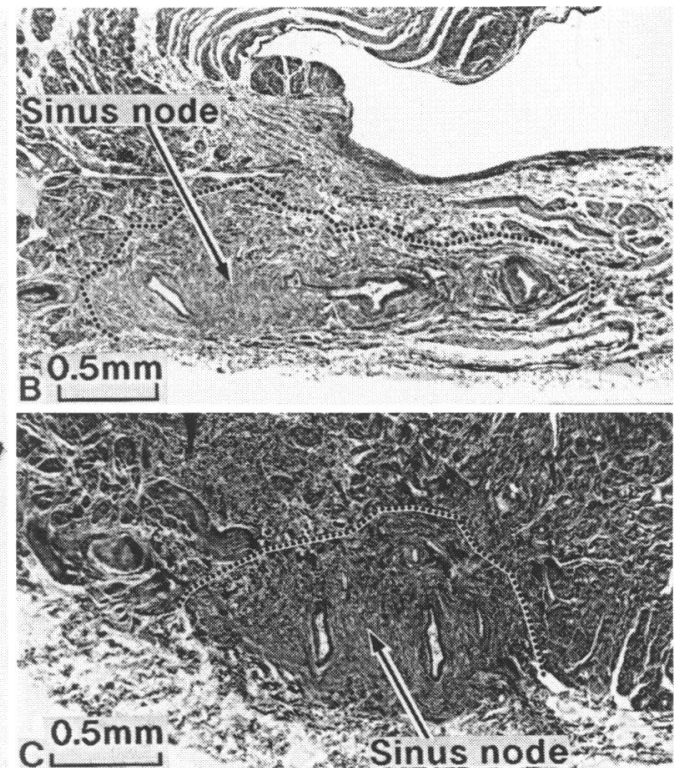

Figure 1 Transverse sections through the cavoatrial junction of a fetal heart (18 weeks gestation) with isomerism of the right atrial appendages. Bilateral sinus nodes (within dotted lines), represented by small interweaving cells clustered around the nodal arteries are shown in panels $B$ and $C$.
Figure $2 A$ and $B$ are right and left views respectively of a fetal heart of 12 week gestation. The asterisk indicates the position of the hypoplastic sinus node as shown in panel $C$.
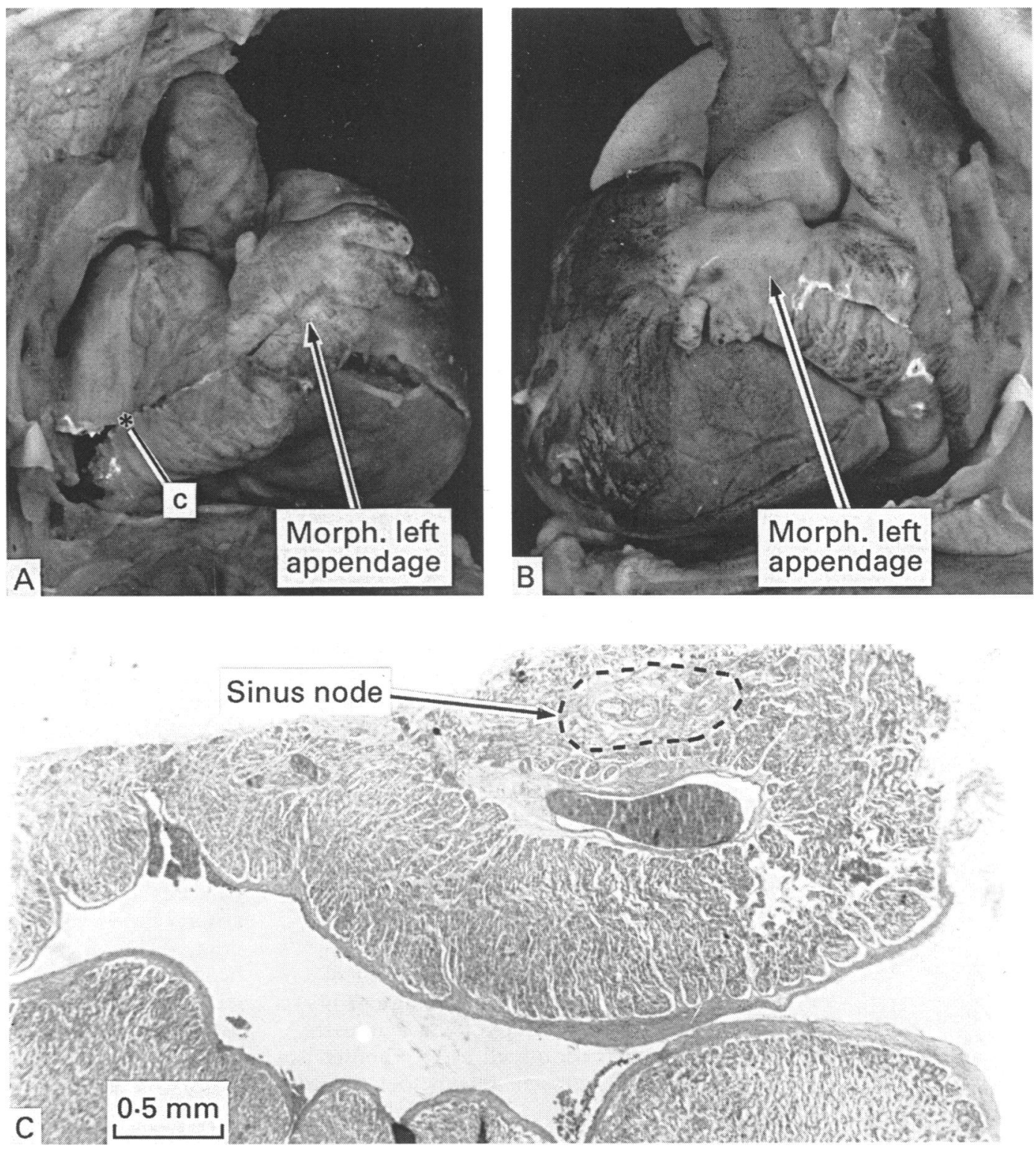


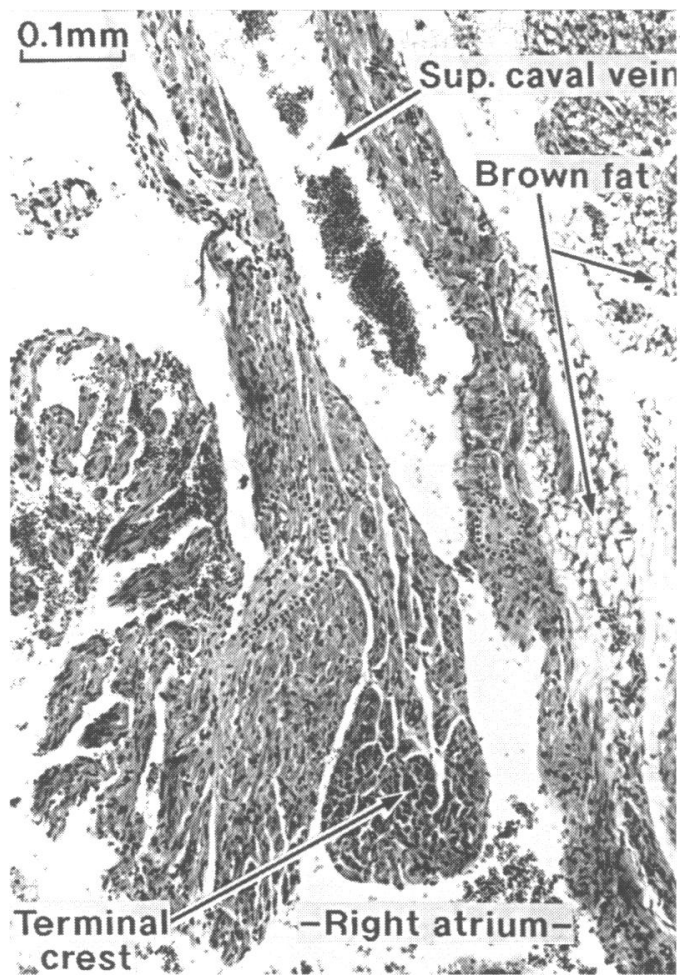

Figure $3 A$ section in the coronal plane of an adult mouse heart with usual atrial arrangement shows the entrance of the superior caval vein into the right atrium. The sinus node (within dotted lines) is seen as a collection of spindle cells around a central artery at the junction between right appendage and the superior caval vein. The node partly encircles the vein to reach to the posterior side of the atrium where there are lobules of brown fat.

studied. There were five adults, 18 fetuses at 18 or 19 days gestation, and two neonates less than two days of age. As in the human hearts, right isomerism was recognised on the basis of the bilateral presence of broad appendages and terminal crests with pectinate muscles extending round the vestibule. The atrial appendages in left isomerism were also broad bilaterally, but were distinguishable from morphologically right appendages by their narrow junctions with the venous atrial components and the absence of pectinate muscles round the vestibule. Four hearts were identified as having isomerism of the right atrial appendages, while nine hearts had isomerism of the left atrial appendages. The remaining 12 hearts, with usual or mirror imaged arrangement of the atrial appendages, were used to determine the normal morphology of the sinus node in the iv/iv mouse.

All heart specimens were fixed in formaldehyde and then processed in their entirety for histological sectioning, blocking them in paraffin wax for serial sectioning at $10 \mu \mathrm{m}$ thickness. The sections were collected on cardboard trays and numbered sequentially. Every 25th section of the human hearts, and every fifth section from the mouse hearts, was mounted on glass slides and stained with a modified Masson's trichrome technique. ${ }^{9}$ All the stained sections were examined and additional sections mounted and stained where necessary. The sinus node was identified in the human hearts using well established histological criteria. ${ }^{41011}$ For the mouse hearts, the controls with lateralised atrial arrangement

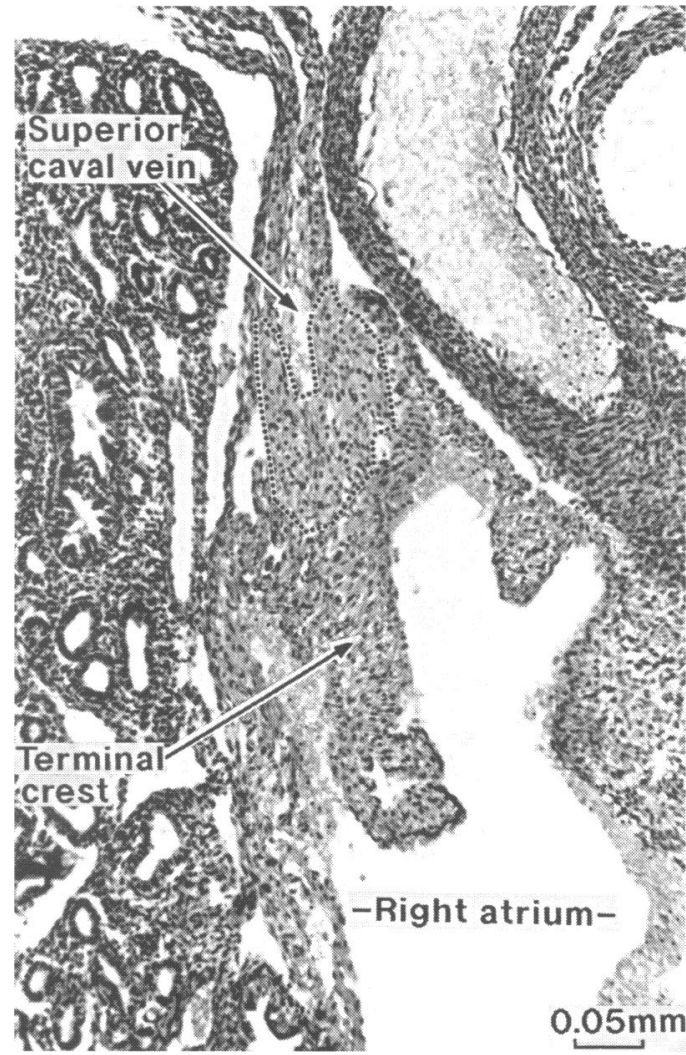

Figure 4 The heart of a normal fetal mouse sectioned in a coronal plane. The sinus node (within dotted line) is horseshoe shaped and draped across the junction between the superior caval vein and the right atrium. The nodal cells are less darkly stained than ordinary myocytes.

were used to determine the histological characteristics of the sinus nodes.

\section{Results}

HUMAN HEARTS

Isomerism of the right atrial appendages

There were bilateral superior caval veins in these two hearts. Bilateral sinus nodes were identified in each heart. The nodes were recognised by their composition of small interweaving cells within a fibrous matrix, usually surrounding a small artery. ${ }^{4}$ The nodal cells stained slightly less intensely than the surrounding atrial myocytes. In one heart, each node was spindle shaped and located subepicardially in the terminal groove, lateral to the cavoatrial junction (fig 1). In the other heart, the sinus node on the right side was normally located and supplied by an artery which passed through its centre. The node on the left was hypoplastic and situated more inferiorly and posteriorly than usual along the terminal groove. It was recognised by its composition of small cells and fibrous network around an artery.

\section{Isomerism of the left atrial appendages}

The sinus node was identified as a recognisable structure in only three of the 12 hearts. In one case, the node was well formed and situated on the crest of the right sided atrial appendage (of left morphology) but in the 


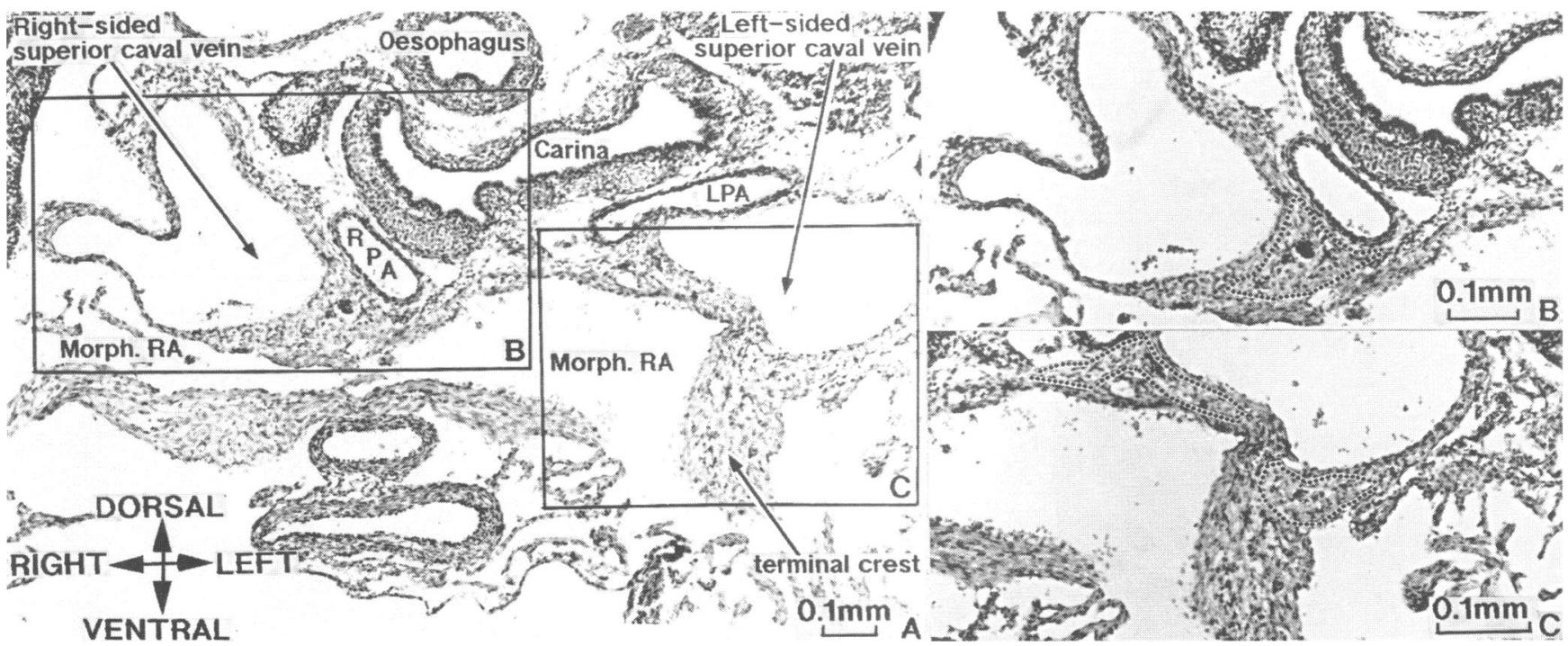

Figure 5 The thorax of a fetal mouse (18 day gestation) with isomerism of the right atrial appendages is sectioned in a transverse plane. Enlargements of the boxed areas $(B$ and $C$ ) show sinus nodes (within dotted lines) represented by vacuolated cells around the cavoatrial junctions on both sides. $L P A$, left pulmonary artery; $R A$, right atrium; $R P A$, right pulmonary artery.

absence of a superior caval vein. In the second case, the node was hypoplastic, represented by a tiny cluster of small cells around a prominent nodal artery. It was located in the posterior wall of the right sided atrium, inferior to its junction with the superior caval vein. The recognisable sinus node in the third case was also hypoplastic and situated at the posterior junction between the right sided atrium and the right superior caval vein (fig 2). Bilateral caval veins were present in this case.

\section{MOUSE HEARTS}

Usual and mirror image arrangement of the atrial appendages

The sinus node was located in subepicardial position at the upper part of the junction of the terminal groove with the superior caval vein in the morphologically right atrium. In contrast to the usual lateral position in human hearts, the sinus node in the murine heart was more extensive and straddled the crest of the morphologically right appendage (fig 3 ). In keeping with this, the node was related to the left sided appendage in hearts with mirror image arrangement of the atrial appendages. The node in the fetus was relatively bigger than the node in the adult. In the fetus, it partly or almost encircled the junction of the superior caval vein with the morphologically right atrium (fig 4). Histologically, the node was composed of compactly arranged, pale staining, non-striated short cells which were not noticeably smaller in diameter than ordinary atrial myocytes. Unlike the situation in the human, the nodal cells were not arranged within a prominent fibrous tissue matrix. The nodal cells in the fetal hearts were slightly bigger than ordinary atrial myocytes and their nuclei and cytoplasm were vacuolated. Collections of ganglion cells were frequently found close to the nodes.

\section{Isomerism of the right atrial appendages}

Two distinct bilateral sinus nodes were found in each case. They were bigger than usual, occupying a transmural position at the lower end of the superior caval vein at its junction

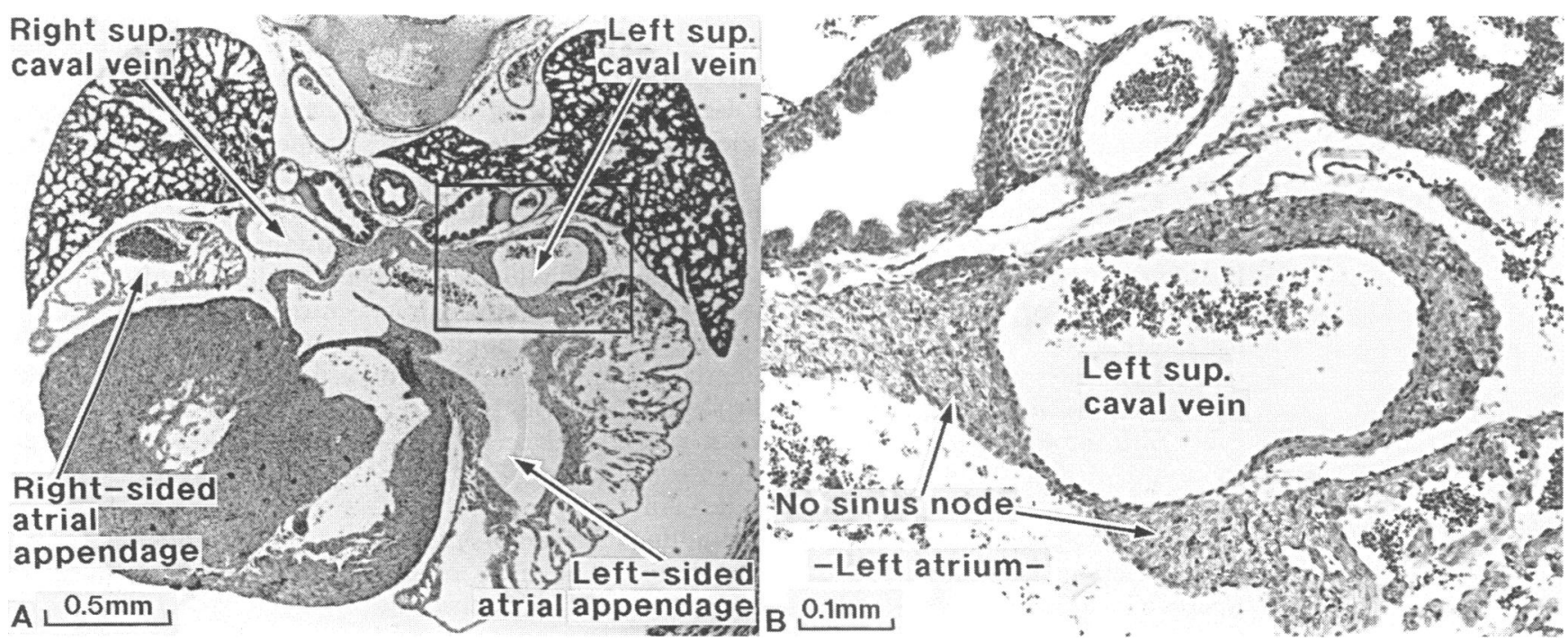

Figure 6 A transverse section through the thorax of a fetal mouse (19 day gestation) with left isomerism. Collections of vacuolated cells are lacking on both sides. The left cavoatrial junction is enlarged in panel $B$. 


\section{A Usual atrial arrangement}

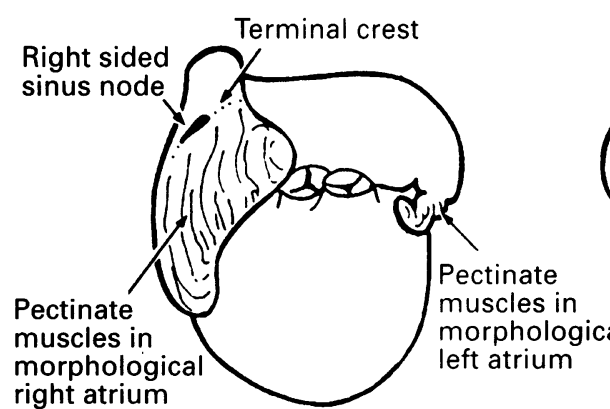

C Right isomerism

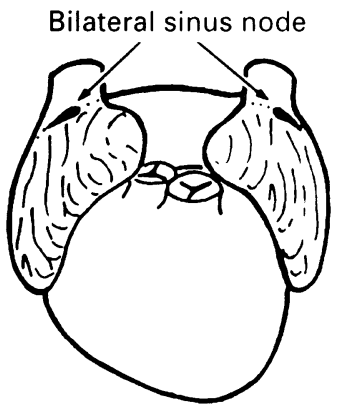

B Mirror imaged atrial arrangement

Left sided

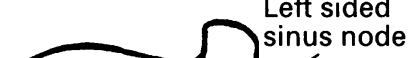

specimens $^{1213}$ and animal models ${ }^{1617}$ which showed that recognition of the morphology of each appendage depended on the nature of its junction with the rest of the atrial chamber, rather than relying merely on its size and shape, or upon the venoatrial connections. The ultimate difference between the two types of atria, therefore, still remains to be determined. At the microscopic level, evidence to date suggests that the presence or absence of a sinus node ${ }^{1}$ could be the most recognisable distinction between the two types of atrial appendage. With this in mind, we have studied the location and morphology of the sinus node in both human and mouse hearts in which isomerism of the atrial appendages had been diagnosed grossly, as judged from morphological criteria. Our objectives were to examine the usefulness of recognising the presence of the sinus node in the determination of atria of right morphology, and its absence in atria of left morphology, and to reevaluate the concept of isomerism in terms of its functional significance.

Our material, therefore, included hearts with isomerism of right or left types from human fetuses and iv/iv mice. Our earlier studies offer evidence that the arrangement in the human fetus can be generalised into the situation in childhood and adulthood. ${ }^{4}$ The experimental model of our $i v / i v$ mice, however, needed to be assessed further to establish its validity in this context. The $i v / i v$ mouse is a model of abnormal laterality which has been said to show mirror image arrangement in approximately half of adult animals. ${ }^{18}$ More critical examination, nonetheless, has shown that many iv/iv mice show so called heterotaxy. ${ }^{161719-21}$ Our previous study on $i v / i v$ mice, for example, showed that $1.5 \%$ and $3.3 \%$ had right and left isomerism, respectively. ${ }^{7}$ Morphologically right and left atrial appendages could be distinguished using criteria very similar to those applied on human hearts. The sinus nodes in our control group of mouse hearts were also histologically recognisable structures, consistent with previous descriptions. ${ }^{22}$ We are not aware of any previous study on the sinus node of murine hearts with isomeric arrangements of the atrial appendages.

On studying our human and murine hearts, we discovered bilateral sinus nodes in all hearts with isomeric arrangement of morphologically right appendages, while a single sinus node was observed in the superior cavoatrial junction of each morphologically right atrium in hearts with lateralised arrangement (fig 7). Our study has shown, therefore, that the morphologically right appendage is accompanied by a sinus node irrespective of its location. The node was almost always found at the junction of the superior caval vein with the appendage, albeit that one node was displaced posteriorly and inferiorly along this junction in a heart with isomerism of the morphologically right appendages. Clinical studies on atrial excitation in hearts with isomerism of right atrial appendages have also shown the presence of bilateral sinus nodes, ${ }^{2324}$ while the hiscategorised into partial forms of these lateralised patterns. ${ }^{31415}$

This latter concept, however, has ignored evidence from earlier studies in both human 
tological proof of this gross finding has long been established by Van Mierop and Wiglesworth. ${ }^{1}$ Taken together, these findings support the notion that the junctions of the appendages with the rest of the atrium are truly isomeric in those hearts we have characterised grossly as showing bilateral right appendages. This is also supported by the consistent association of other cardiac lesions, such as the absence of the atrial septum and the coronary sinus, and abnormal pulmonary venous connections. ${ }^{125}$

Bilateral absence of the sinus node was a feature in most hearts with isomerism of the morphologically left appendages. In three cases, however, a small nodal replica was discovered in an abnormal position at the postero-inferior wall of the right sided morphologically left atrium, while in one case the node was located more superiorly. Absence, or virtual absence, of the sinus node can therefore be taken as a marker of the morphologically left atrium. Clinical studies on patients with left isomerism have shown the presence of a $P$ wave which was almost always abnormal in its axis. ${ }^{23} 2426$ The abnormal location of a hypoplastic node, or absence of the node, is an attractive morphological finding with which to explain this abnormal $P$ wave.

Our morphological criteria on the recognition of the atrial arrangements are useful for anticipating the functional activity of the sinus node, and conversely have shown that isomerism of the atrial appendages exists in conjunction with the arrangement of the sinus node. If there is a narrow tubular zone between the venous component and the appendage, with pectinate muscles confined to the appendages, the atrium is of left morphology and no well formed specialised junctional node is expected. In the morphologically right atrium, in contrast, the transition from the superior caval vein to the appendage is marked externally by the terminal groove, which corresponds to the terminal crest on the endocardial aspect. The sinus node can be anticipated in the terminal groove, even in the absence of a superior caval vein. ${ }^{27}$ Further studies on the processes involved in development of atria of left or right morphology will provide more understanding of the differences between right and left morphologies, as well as those between right and left isomerism.

1 Van Mierop LHS, Wiglesworth FW. Isomerism of the cardiac atria in the asplenia syndrome. Lab Invest 1962;11: 1303-15.
2 Becker AE, Anderson RH. Isomerism of the atrial appendages - goodbye to asplenia and all that. In: Clark $\mathrm{EB}$, Takao A, eds. Developmental cardiology: morphogenesis and functions. Mount Kisco: Futura Publishing Co, 1990:659-70.

3 Van Praagh R, Van Praagh S. Atrial isomerism in the heterotaxy syndromes with asplenia, or polysplenia, or normally formed spleen: an erroneous concept. $A m \mathcal{F}$ Cardiol 1990;66:1504-6.

4 Anderson KR, Ho SY, Anderson RH. The location and vascular supply of the sinus node in the human heart. $\mathrm{Br}$ veart $₹ 1979 ; 41: 28-52$.

5 Mandel WJ, Lak MM, Obayashi K. Sinus node function evaluation in patients with and without sinus node disease. Arch Intern Med 1975;135:388-92.

6 Ho SY, Cook A, Anderson RH, Allan LD, Fagg N. Isomerism of the atrial appendages in the fetus. Pediat Pathol 1991;11:589-608.

7 Seo JW, Brown NA, Ho SY, Anderson RH. Abnormal laterality and congenital cardiac anomalies: relationships of visceral and cardiac morphologies in the iv/iv mouse. Circulation 1992;67:316-20.

8 Ho SY, Fagg N, Anderson RH, Cook A, Allan I. Disposition of the atrioventricular conduction tissues in hearts with isomerism of the atrial appendages its rela hearts with isomerism of the atrial appendages - its relationship to congenitally

9 Smith A, Ho SY, Anderson RH. Histologic study of the cardiac conducting system as a routine procedure. Med cardiac conducting system

10 James TN. Anatomy of the human sinus node. Anat $R e$ 1961;141:109-16.

11 Truex RC, Smythe MQ, Taylor MJ. Reconstruction of the human sinoatrial node. Anat Rec 1967;159:371-8.

2 Macartney FJ, Zuberbuhler JR, Anderson RH. Morphological considerations pertaining to recognition of atria isomerism: consequences for sequential chamber localisation. Br Heart f 1980;44:657-67.

13 Sharma S, Devine W, Anderson RH, Zuberbuhler JR. The determination of atrial arrangement by examination of Heart $₹ 1988 ; 60: 227-31$.

14 Van Praagh S, Kreutzer J, Alday L, Van Praagh R Systemic and pulmonary venous connections in visceral heterotaxy, with emphasis on the diagnosis of the atrial situs: a study of 109 postmortem cases. In: Clark EB Takao A, eds. Developmental cardiology: morphogenesis and function. Mount Kisco: Futura Publishing Co, 1990 671-727.

15 Van Praagh R, Santini F, Geva T. Segmental situs in con genital heart disease: a fundamental concept. G Ital Cardiol 1990;20:246-53.

16 Icardo JM. Development of the outflow tract: a study in hearts with situs solitus and situs inversus. Ann N Y Acad Sci 1990;588:26-40.

17 Icardo JM, Sanchez de Vega MJ. Spectrum of heart malformations in mice with situs solitus, situs inversus, and associated visceral heterotaxy. Circulation 1991:84: $2547-58$

18 Hummel KP, Chapman DB. Visceral inversion and associated anomalies in the mouse. $\mathcal{F}$ Hered 1959;50:9-13.

19 Layton WM. Random determination of a developmental process: reversal of normal visceral asymmetry in the process: reversal of normal vice

20 Layton WM. Heart malformations in mice homozygous for a gene causing situs inversus. Birth Defects 1978;14: $277-93$.

21 Layton WM. The biology of asymmetry and the development of the cardiac loop. In: Ferrans VJ, ed. Cardia morphogenesis. Amsterdam: Elsevier, 1985:134-40.

22 Lev M, Thaemert JC. The conduction system of the mouse heart. Acta Anat 1973;85:342-52.

23 Dickinson DF, Wilkinson JL, Anderson KR, Smith A, Ho SY, Anderson RH. The cardiac conduction system in situs ambiguus. Circulation 1979;59:879-85.

24 Wren C, Macartney FJ, Deanfield J. Cardiac rhythm in atrial isomerism. Am $\mathcal{f}$ Cardiol 1987;59:1156-8.

25 Rose V, Izukawa T, Moes CAF. Syndromes of asplenia and polysplenia. A review of cardiac and non-cardiac malformations in 60 cases with special reference to diagnosis mations in 60 cases with special reference
and prognosis. Br Heart 7 1975;37:840-52.

26 Momma K, Takao A, Shibata. Characteristics and natural history of abnormal atrial thythms in left isomerism. $A m \mathcal{F}$ history of abnormal atrial

27 Lenox CC, Hashida Y, Anderson RH, Hubbard JD Conduction tissue anomalies in absence of the righ superior caval vein. Int $\mathcal{F}$ Cardiol 1985;8:251-60. 